\title{
Perfil nutricional e dietético de pacientes com hepatite C: uma revisão integrativa da literatura
}

\author{
Nutritional and dietary profile of patients with hepatitis $C$ : an \\ integrative literature review
}

\author{
Tauana da Costa Alves ${ }^{1}$, Leucinéia Schmidt ${ }^{1}$, Fábia Benetti ${ }^{1}$
}

1- Universidade Regional Integrada do Alto Uruguai e das Missões - URI, Frederico Westphalen, RS, Brasil.

\begin{abstract}
RESUMO
Introdução: o estado nutricional e o perfil dietético são considerados fatores importantes no tratamento e prevenção da hepatite C. Objetivo: revisar a literatura sobre o estado nutricional e o perfil dietético de pacientes com hepatite C. Método: trata-se de uma revisão integrativa da literatura realizada nas benetti@uri.edu.br seguintes bases de dados: PubMed, Medline e Scielo, aplicando-se a combinação dos seguintes descritores: "Hepatite C", "Avaliação Nutricional", "Antropometria" e "Recomendações Nutricionais". Resultados: foram selecionados 10 artigos para o presente estudo, através dos quais constatou-se que não se tem ainda um padrão ouro para avaliar o estado nutricional de pacientes com hepatite $\mathrm{C}$, e por isso são utilizados diversos métodos combinados como: antropometria (peso, altura, índice de massa corporal, circunferência da cintura, dobras cutâneas, circunferência braquial, circunferência muscular do braço), bioimpedância, avaliação subjetiva global, avaliação da força de preensão palmar, métodos bioquímicos e anamnese alimentar. Quanto à classificação do estado nutricional de pacientes com hepatite pode-se perceber que alguns estudos trazem a classificação de sobrepeso e excesso de gordura abdominal em detrimento de outros estudos que demonstram a prevalência de desnutrição em estágios mais avançados da doença. Ainda, vários estudos demonstraram irregularidades em relação à ingestão de energia, macro e micronutrientes. Considerações finais: embora existam vários métodos

Palavras-chave:

Hepatite C; Avaliação

empregados na avaliação nutricional de pacientes com hepatite $\mathrm{C}$, classificar o estado nutricional dos mesmos ainda é um desafio. São necessários mais estudos e validação de protocolos específicos para a avaliação nutricional ser mais precisa e assertiva. Além disso, é necessário monitorar frequentemente a ingestão dietética destes pacientes.
\end{abstract} Nutricional; Antropometria; Recomendações Nutricionais.

\begin{abstract}
Introduction: nutritional status and dietary profile are considered important factors in the treatment and prevention of hepatitis C. Objective: to review the literature on the nutritional status and dietary profile of patients with hepatitis C. Method: this is an integrative review of the literature on the following databases: PubMed, Medline, and Scielo, applying a combination of the following descriptors: "Hepatitis C", "Nutritional Assessment", "Anthropometry" and "Recommended Dietary Allowance". Results: a total of 10 articles were selected for the present study, which allowed to verify that there is still no gold standard to evaluate the nutritional status of patients with hepatitis $\mathrm{C}$, and therefore several combined methods are used such as anthropometry (weight, height, body mass index, waist circumference, skinfolds, arm circumference, arm muscle circumference), bioimpedance, global subjective assessment, functional evaluation of palmar grip strength, biochemical methods and food anamnesis. Regarding the classification of nutritional status of patients with hepatitis $\mathrm{C}$, some studies have led to the classification of overweight and excess abdominal fat in detriment of other studies that demonstrate the prevalence of malnutrition in more advanced stages of the disease. Still, several studies have shown irregularities regarding energy intake, and macro and micronutrients. Closing remarks: although there are several methods used in the nutritional evaluation of patients with hepatitis C, classifying their nutritional status is still a challenge. Further studies and validation of specific protocols are needed for nutritional assessment to be more accurate and assertive. In addition, it is necessary to monitor the dietary intake of these patients frequently.
\end{abstract}

Keywords:

Hepatitis C; Nutrition Assessment; Anthropometry; Recommended Dietary Allowance. 


\section{INTRODUÇÃO}

A hepatite $C$ representa uma inflamação do fígado causada pela infecção pelo vírus da hepatite $\mathrm{C}$ (HCV), sendo uma das principais causas de doença hepática crônica, levando ao desenvolvimento de cirrose e carcinoma hepatocelular, o que afeta aproximadamente 185 milhões de pessoas no mundo. ${ }^{1}$ O HCV pode ser adquirido a partir de transfusão de sangue, uso de drogas intravenosas, uso de agulhas e/ou instrumentos cortantes não esterilizados em procedimentos médicos, odontológicos, e tatuagens..$^{2,3}$ A presença de anticorpos anti-HCV pode indicar infecção por hepatite $\mathrm{C}$ atual ou passada e, quando não tratada, a infecção crônica pode chegar a $85 \%{ }^{4}{ }^{4}$

Os sinais e sintomas são comuns às demais doenças parenquimatosas hepáticas e costumam manifestar-se apenas em fases mais avançadas da doença. $\mathrm{O}$ dano hepático em geral promove grande impacto nutricional, independente da etiologia, devido à participação do fígado nos processos digestivos e absortivos, na metabolização e no armazenamento dos nutrientes. ${ }^{5}$

$\mathrm{O}$ aumento no número de casos de pacientes com HCV despertou o interesse em estudar e conhecer o perfil nutricional de pacientes com esta hepatopatia, para prestar assistência e subsidiar a orientação nutricional adequada. ${ }^{6}$

Nesse sentido, a promoção do estado nutricional adequado, a detecção precoce do excesso de peso, o tratamento das deficiências e/ou excesso de nutrientes específicos são objetivos do acompanhamento clínico e nutricional dos indivíduos com hepatite $\mathrm{C}$, haja vista que potencialmente poderá retardar a progressão da doença hepática. ${ }^{6}$

Atualmente, estão disponíveis os seguintes métodos de avaliação nutricional: a) medidas antropométricas, que abrangem: peso, altura, índice de massa corporal (IMC), dobras cutâneas, mais comumente a prega cutânea tricipital (PCT), circunferência braquial(CB) e circunferência muscular do braço (CMB); b) métodos funcionais, que incluem a força muscular, medida através da dinamometria, ou força de aperto da mão não-dominante (FAM) e a espessura do músculo adutor do polegar (MAP); c) métodos bioquímicos, sendo os mais importantes a albumina, pré-albumina, proteína carreadora de retinol e transferrina; d) avaliação nutricional clínica, sendo realizada através da Avaliação Subjetiva Global
(ASG) e anamnese alimentar. ${ }^{7}$

Os resultados de métodos tradicionalmente empregados para a avaliação do estado nutricional podem ser comprometidos pelas alterações corporais e metabólicas decorrentes da cirrose, como modificação na proporção da água corporal, por ascite e edema. ${ }^{8}$ Dessa forma, o IMC acaba não identificando a desnutrição, mas pelo contrário, classificando os pacientes como em estado nutricional de sobrepeso. Tal fato pode ser explicado pela presença de ascite e edema nesses pacientes, fazendo com que apresentem um peso aumentado. ${ }^{7}$

A sarcopenia também é frequente em pacientes com hepatopatias, resultando em atrofia muscular, perda de força e massa muscular, dificultando desta forma a avaliação do estado nutricional e contribuído para a mortalidade destes indivíduos. ${ }^{9}$ Hayashi et al. ${ }^{10}$ relataram que $44,4 \%$ dos homens e $38,2 \%$ das mulheres com doença hepática apresentavam sarcopenia.

Uma alimentação adequada para prevenção e tratamento de hepatopatias pode ser considerada como um importante fator terapêutico. A ingestão apropriada de macro e micronutrientes têm como objetivo corrigir deficiências nutricionais e promover o adequado estado nutricional dos pacientes, de forma a alcançar um equilíbrio positivo de nitrogênio e evitar agentes hepatotóxicos. A adição de complementos alimentares e suplementos nutricionais são indicados conforme a necessidade do indivíduo. As restrições dietéticas podem ser prejudiciais e, portanto, devem ser individualizadas. ${ }^{11}$

Diante do exposto, esta revisão da literatura teve como objetivo demonstrar o perfil nutricional e dietético de pacientes portadores de hepatite $\mathrm{C}$, a fim de melhorar a qualidade de vida dessa população.

\section{MÉTODO}

Trata-se de uma revisão integrativa da literatura, a qual tem como finalidade sintetizar e incluir a análise de pesquisas relevantes, que dão suporte para a tomada de decisão e a melhoria da prática clínica, possibilitando a síntese de informação sobre o assunto, além de apontar lacunas do conhecimento que precisam ser preenchidas com a realização de novos estudos. ${ }^{12}$

O questionamento que norteou o estudo foi: Qual o estado nutricional e o perfil dietético de 
pacientes com hepatite $\mathrm{C}$ ?

Para o levantamento bibliográfico, optou-se pela busca de artigos nas bases de dados PubMed, Medline e Scielo, nos idiomas português e inglês, sem incluir como filtro o recorte temporal dos estudos. Os descritores empregados enquadram-se nos Descritores em Ciências da Saúde (DeCS). Utilizou-se o modo de pesquisa avançada e integrada, com as seguintes palavras: "Hepatite C", "Avaliação Nutricional", "Antropometria" e "Recomendações Nutricionais".

O período destinado à busca de artigos ocorreu de março a abril do ano de 2017. Foram definidos como critérios de inclusão: pesquisas que abordassem a relação existente entre a hepatite $\mathrm{C}$ e o perfil nutricional e dietético destes pacientes, e como critérios de exclusão: trabalhos que não se relacionassem com o tema da pesquisa, não apresentassem resumos na íntegra, teses, dissertações e resumos publicados em anais.

O ano de 2017 foi considerado, entretanto a presente investigação pode não trazer todas as pesquisas da temática de 2017, em virtude de ser o ano referente à realização da pesquisa. Os resumos foram avaliados, e em seguida as produções foram lidas na íntegra.

Para a organização das informações, desenvolveu-se uma planilha contendo: autor, ano e título da pesquisa, indexado (banco de dados onde a pesquisa foi indexada), população, objetivos do estudo, metodologia, principais resultados e conclusões.

\section{RESULTADOS}

Foram encontrados 26 artigos, e após a aplicação dos critérios de inclusão e exclusão foram selecionados 10 artigos para o presente estudo, sobre pacientes com hepatite $C$, que investigaram o estado nutricional e o perfil dietético desses indivíduos.

A partir das informações disponibilizadas nos artigos selecionados, construiu-se o quadro abaixo (Quadro 1), com o intuito de sintetizar os principais achados sobre o tema em questão.

Quadro 1 - Descrição de estudos sobre perfil nutricional e dietético de pacientes com hepatite C, indexados no Pubmed, Medline e Scielo.

\begin{tabular}{|c|c|c|c|c|c|}
\hline $\begin{array}{l}\text { Autor, ano e } \\
\text { título }\end{array}$ & Indexado & População & $\begin{array}{c}\text { Objetivos do } \\
\text { estudo }\end{array}$ & Metodologia do estudo & Resultados \\
\hline $\begin{array}{l}\text { Carreira e } \\
\text { Pereira(2011).6 } \\
\text { Nutritional and } \\
\text { Dietetic Profile } \\
\text { of individuals } \\
\text { with hepatitis C. }\end{array}$ & Scielo & $\begin{array}{l}82 \\
\text { participantes, } \\
\text { sendo } 56 \text { com } \\
\text { hepatite C,e } \\
\text { a maioria } \\
\text { do sexo } \\
\text { masculino } \\
(76,8 \%) .\end{array}$ & $\begin{array}{l}\text { Avaliar } \\
\text { o estado } \\
\text { nutricional } \\
\text { e a ingestão } \\
\text { dietética de } \\
\text { pacientes com } \\
\text { hepatite C. }\end{array}$ & $\begin{array}{l}\text { Estudo observacional, em que os } \\
\text { indivíduos foram selecionados } \\
\text { aleatoriamente e aqueles que } \\
\text { concordaram em participar realizaram } \\
\text { os exames laboratoriais necessários } \\
\text { e foram agendados para consulta } \\
\text { nutricional. Os participantes } \\
\text { foram distribuídos em três grupos } \\
\text { amostrais: G1 indivíduos saudáveis, } \\
\text { G2indivíduos com hepatite C sem } \\
\text { tratamento antiviral, e G3 indivíduos } \\
\text { com hepatite C em tratamento } \\
\text { antiviral. O estado nutricional foi } \\
\text { avaliado de acordo com o IMC e o } \\
\text { percentual de gordura corpórea foi } \\
\text { obtido mediante a somatória das } 4 \\
\text { dobras cutâneas (bicipital, tricipital, } \\
\text { subescapular e supra ilíaca). A análise } \\
\text { dietética foi realizada por meio do } \\
\text { cálculo do recordatório alimentar de } \\
\text { três dias consecutivos e os resultados } \\
\text { da ingestão média diária de energia, } \\
\text { proteína, carboidrato, lipídio e } \\
\text { fibras foram comparados com as } \\
\text { recomendações da National Academy } \\
\text { of Sciences. }\end{array}$ & $\begin{array}{l}\text { Os indicadores } \\
\text { antropométricos mostraram } \\
\text { que a média de peso e } \\
\text { porcentagem de gordura } \\
\text { corpórea do G1 e G2 e o } \\
\text { IMC do G2 e G3 estavam } \\
\text { aumentados. A ingestão } \\
\text { energética e proteica estavam } \\
\text { adequadas. O consumo } \\
\text { médio de carboidratos foi } \\
\text { acima do recomendado e } \\
\text { a porcentagem média de } \\
\text { lipídeos foi adequada, mas o } \\
\text { G2 consumiu mais lipídeos } \\
\text { e fibras que o G3, sendo } \\
\text { estatisticamente diferentes; } \\
\text { entretanto a quantidade de } \\
\text { fibras ingeridas foi abaixo do } \\
\text { recomendado nos três grupos. }\end{array}$ \\
\hline
\end{tabular}




\begin{tabular}{|c|c|c|c|c|}
\hline $\begin{array}{l}\text { Ismail et } \\
\text { al.(2012). } \\
\text { Nutritional status } \\
\text { in patients with } \\
\text { hepatitis C. }\end{array}$ & PubMed & $\begin{array}{l}\text { Participaram } \\
\text { do estudo } 400 \\
\text { pessoas. }\end{array}$ & $\begin{array}{l}\text { Avaliar o estado } \\
\text { nutricional } \\
\text { através daASG. }\end{array}$ & $\begin{array}{l}\text { Foram selecionados } 400 \\
\text { participantes, } 100 \text { pertenciam } \\
\text { ao grupo G1 (Controle } \\
\text { Saudáveis), } 100 \text { ao grupo } \\
\text { G2 (pacientes com hepatite } \\
\text { C), } 100 \text { eram do grupo G3 } \\
\text { (Cirróticos Compensados) } \\
\text { e } 100 \text { eram do grupo G4 } \\
\text { (Cirróticos Descompensados). } \\
\text { Utilizou-se a ASG como } \\
\text { instrumento para avaliar } \\
\text { o estado nutricional dos } \\
\text { pesquisados. }\end{array}$ \\
\hline
\end{tabular}

A maioria dos pacientes do grupo G1 foram classificados como bem nutridos (classe A) segundo a ASG. No grupo G2 14\% dos pacientes foram classificados como moderadamente desnutridos (classe B). No grupo G3 de cirróticos compensados a maioria $90 \%$ estavam desnutridos. E no grupo G4 a maioria dos pacientes (98\%) foram classificados como gravemente desnutridos (classe C). Desta forma, verificou-se que à medida que a doença hepática se agrava maior é o risco de depleção do estado nutricional.

A desnutrição foi correlacionada com a gravidade clínica da doença hepática. A maioria dos pesquisados (64\%) desenvolveram a doença hepática pelo uso de álcool, seguidos de $25 \%$ por hepatite C. Quanto ao estado nutricional 16 pacientes apresentaram desnutrição grave, 7 com etiologia alcoólica (43\%), 8 com etiologia viral (50\%) e 1 com etiologia criptogênica (7\%). Todos os pacientes possuíam encefalopatia ou ascite moderada/ grave. A desnutrição proteicocalórica pode ser identificada em todos os estágios clínicos, mas foi mais prevalente em estágios avançados da doença hepática. A presença de desnutrição está associada ao aumento do número de complicações e mortalidade a curto e longo prazo.

\begin{tabular}{|c|c|c|c|}
\hline $\begin{array}{l}\text { Fioravante et } \\
\text { al.(2012). }{ }^{20} \\
\text { Weight loss and } \\
\text { resting energy } \\
\text { expenditure in } \\
\text { patients with } \\
\text { chronic hepatitis } \\
\text { C before and } \\
\text { during standard } \\
\text { treatment. }\end{array}$ & PubMed & $\begin{array}{l}42 \text { pacientes } \\
\text { com hepatite } \\
\text { C. }\end{array}$ & $\begin{array}{l}\text { Avaliar o estado } \\
\text { nutricional, } \\
\text { composição } \\
\text { corporal e gasto } \\
\text { energético de } \\
\text { repouso em } \\
\text { pacientes com } \\
\text { hepatite C } \\
\text { antes e durante } \\
\text { o tratamento } \\
\text { com Interferon } \\
\text { Peguilado e } \\
\text { Ribavirina. }\end{array}$ \\
\hline
\end{tabular}

\begin{tabular}{llll}
\hline Port et al.(2014). ${ }^{10}$ & Scielo & Foram & Identificar \\
Biochemical & & entrevistados & alterações no \\
nutritional profile & & 31 pacientes & metabolismo \\
of liver cirrhosis & com HCV e & de macro e \\
patients with & carcinoma & micronutrientes \\
hepatocellular & hepatocelular & entre pacientes \\
carcinoma. & e 48 pacientes & com cirrose \\
\hline
\end{tabular}

Estudo prospectivo que avaliou pacientes com hepatite $\mathrm{C}$ antes e após 12 semanas de tratamento com Interferon Peguilado e Ribavirina. A avaliação nutricional consistiu-se de antropometria (peso, altura, IMC e circunferência da cintura) e a composição corporal foi determinada através da bioimpedância. $\mathrm{O}$ Gasto Energético de Repouso (EER) de cada indivíduo foi obtido por calorimetria indireta.

Estudo transversal e analítico.

\section{Foram realizados exames} laboratoriais e avaliada a existência de associação entre os parâmetros bioquímicos e a gravidade da doença, bem como a presença de
Houve perda significativa de peso durante o tratamento com consequente diminuição do IMC, acompanhado de diminuição significativa da massa magra. No entanto, não houve alteração no EER, mas é necessária uma intervenção para diminuir possíveis danos. 


\begin{tabular}{|lll}
\hline $\begin{array}{l}\text { com cirrose } \\
\text { hepática. }\end{array}$ & $\begin{array}{l}\text { hepática com e } \\
\text { sem carcinoma } \\
\text { hepatocelular. }\end{array}$ & $\begin{array}{c}\text { carcinoma hepatocelular. } \\
\text { colesterol e triglicerídeos), } \\
\text { proteínas (albumina, creatinina e } \\
\text { ácido úrico), ferro (transferrina } \\
\text { e ferritina), hematócrito, } \\
\text { hemoglobina, zinco e vitamina } \\
\text { B12. Observou-se que a } \\
\text { desnutrição é frequente em } \\
\text { pacientes com hepatite C, } \\
\text { pois a prevalência de ingestão } \\
\text { inadequada de energia, proteínas } \\
\text { e micronutrientes é comum nessa } \\
\text { população. }\end{array}$ \\
\end{tabular}

\begin{tabular}{|c|c|c|c|c|}
\hline $\begin{array}{l}\text { Gottschall et } \\
\text { al.(2015). }{ }^{26} \\
\text { Nutritional status } \\
\text { and dietary } \\
\text { intake in non- } \\
\text { cirrhotic adult } \\
\text { chronic hepatitis c } \\
\text { patients. }\end{array}$ & Scielo & $\begin{array}{l}94 \text { pacientes } \\
\text { ambulatoriais } \\
\text { ( } 30 \text { a } 76 \\
\text { anos), } \\
\text { sendo } 46 \\
\text { homens. }\end{array}$ & $\begin{array}{l}\text { Aplicar diferentes } \\
\text { métodos de } \\
\text { avaliação do } \\
\text { estado nutricional } \\
\text { de pacientes } \\
\text { adultos com } \\
\text { hepatite C } \\
\text { e descrever } \\
\text { inadequações na } \\
\text { ingestão dietética. }\end{array}$ & $\begin{array}{l}\text { Estudo transversal com } \\
\text { pacientes portadores do vírus } \\
\text { da hepatite C não cirróticos } \\
\text { e não transplantados } \\
\text { em acompanhamento } \\
\text { ambulatorial no Hospital de } \\
\text { Clínicas de Porto Alegre. A } \\
\text { avaliação nutricional incluiu } \\
\text { antropometria (peso, altura, } \\
\text { IMC, PCT, CB, CMB e área } \\
\text { muscular do braço), ASG, } \\
\text { Avaliação Subjetiva do Royal } \\
\text { Free Hospital, dinamometria } \\
\text { e Recordatório de } 24 \text { horas } \\
\text { (R24h). }\end{array}$ \\
\hline
\end{tabular}

Encontrou-se prevalência de desnutrição, a qual é frequente em pacientes com hepatite $\mathrm{C}$, devido à ingestão inadequada de macro e micronutrientes, mesmo na ausência de cirrose.

Santis e Silva et Scielo al.(2015). ${ }^{11}$

Association of the nutritional profile

with histological

findings of

patients with

genotype 1

chronic hepatitis c

infection.

\begin{tabular}{|c|c|c|}
\hline $\begin{array}{l}70 \text { pacientes } \\
\text { com hepatite } \\
\text { C. }\end{array}$ & $\begin{array}{l}\text { Avaliar o estado } \\
\text { nutricional e o } \\
\text { perfil dietético } \\
\text { de pacientes } \\
\text { com hepatite C } \\
\text { crônica e sua } \\
\text { associação com } \\
\text { os achados da } \\
\text { histopatologia. }\end{array}$ & $\begin{array}{l}\text { Foram analisados os seguintes } \\
\text { aspectos: biópsia hepática, } \\
\text { medidas antropométricas } \\
\text { (IMC, circunferência da } \\
\text { cintura, CB, CMB, área } \\
\text { muscular do braço, PCT e } \\
\text { prega cutânea subescapular) } \\
\text { e análise qualitativa e } \\
\text { quantitativa do consumo } \\
\text { alimentar através do R24h. }\end{array}$ \\
\hline
\end{tabular}

A maioria dos pacientes apresentavam obesidade (34\%) ou sobrepeso $(20 \%)$ de acordo com o IMC e risco para doenças cardiovasculares de acordo com a circunferência da cintura (79\%).

$\mathrm{Na}$ análise qualitativa do consumo alimentar, 59\% apresentavam uma dieta inadequada. Verificou-se consumo excessivo de gordura saturada, que esteve associado à esteatose hepática.

\section{Bruch et \\ al.(2016). ${ }^{21}$ \\ Reduced hand \\ grip strength in \\ overweight and \\ obese chronic \\ hepatitis $\mathrm{C}$ \\ patients.}

Scielo

$\begin{array}{ll}\text { 58 pacientes } & \text { Avaliar o estado } \\ \text { com hepatite } & \text { nutricional } \\ \text { C, não } & \text { e o risco } \\ \text { cirróticos } & \text { cardiovascular } \\ \text { e que não } & \text { em pacientes com } \\ \text { estavam sob } & \text { hepatite } \mathrm{C} \text {. } \\ \text { tratamento } & \\ \text { farmacológico } & \\ \text { ativo. } & \end{array}$

Os pacientes foram submetidos à avaliação nutricional através de medidas antropométricas (IMC, circunferência da cintura, $\mathrm{CB}, \mathrm{PCT}$ e circunferência do pescoço) e avaliação funcional, através da medida da força de preensão palmar pela dinamometria. O nível de atividade física foi avaliado através do Questionário Internacional de Atividade Física. Os pacientes também foram submetidos à avaliação clínica e laboratorial. O risco cardiovascular foi calculado pelo escore de Framingham.
A média de idade dos pacientes foi de 51,6 \pm 9,7 anos, sendo 55,2\% do sexo feminino. A prevalência de sobrepeso/obesidade considerando o IMC foi de $70,7 \%$. No entanto, $57,7 \%$ dos homens e $68,8 \%$ das mulheres foram considerados desnutridos de acordo com a força de preensão palmar. Quase 60\% dos pacientes foram considerados sedentários e em relação ao risco cardiovascular, $50 \%$ dos pacientes apresentaram alto risco de sofrer um evento cardiovascular em 10 anos. 


\begin{tabular}{|c|c|c|c|c|c|}
\hline $\begin{array}{l}\text { Pinto et al. } \\
(2016))^{23} \\
\text { Nutritional } \\
\text { status and food } \\
\text { intake of HCV/ } \\
\text { HIV coinfected } \\
\text { patients. }\end{array}$ & PubMed & $\begin{array}{l}57 \text { pacientes } \\
(59,6 \% \\
\text { mulheres }) \\
\text { com idade } \\
\text { média de } 46 \pm \\
11,2 \text { anos. }\end{array}$ & $\begin{array}{l}\text { Avaliar o estado } \\
\text { nutricional, } \\
\text { a ingestão } \\
\text { energética e de } \\
\text { macronutrientes } \\
\text { em pacientes } \\
\text { coinfectados com } \\
\text { HCV/HIV. }\end{array}$ & $\begin{array}{l}\text { O presente estudo avaliou } \\
\text { pacientes cronicamente } \\
\text { infectados com HCV/HIV. } \\
\text { O estado nutricional foi } \\
\text { avaliado através do IMC, } \\
\text { circunferência da cintura, } \\
\text { CB, CMB, PCT e força de } \\
\text { preensão palmar. A ingestão } \\
\text { dietética foi avaliada pelo } \\
\text { R24h. }\end{array}$ & $\begin{array}{l}\text { Identificou-se uma alta prevalência } \\
\text { de sobrepeso e obesidade em } \\
\text { pacientes infectados com HCV/ } \\
\text { HIV. Esses resultados podem estar } \\
\text { relacionados à ingestão dietética } \\
\text { inadequada, a qual foi observada } \\
\text { pelo R24h. }\end{array}$ \\
\hline $\begin{array}{l}\text { Nunes et } \\
\text { al.(2016). }{ }^{25} \\
\text { Food } \\
\text { consumption of } \\
\text { cirrhotic patients, } \\
\text { comparison with } \\
\text { the nutritional } \\
\text { status and disease } \\
\text { staging. }\end{array}$ & Scielo & $\begin{array}{l}25 \text { pacientes } \\
\text { adultos, } \\
\text { cirróticos, } \\
\text { com idade } \\
\text { média de } \\
55,8 \pm 10,8 \\
\text { anos, sendo } \\
56 \% \text { dos } \\
\text { indivíduos } \\
\text { do sexo } \\
\text { masculino. }\end{array}$ & $\begin{array}{l}\text { Avaliar a } \\
\text { ingestão dietética } \\
\text { de pacientes } \\
\text { cirróticos } \\
\text { através do R24h, } \\
\text { correlacionando } \\
\text { com o estado } \\
\text { nutricional e } \\
\text { o estágio da } \\
\text { doença. }\end{array}$ & $\begin{array}{l}\text { Avaliou-se o estado } \\
\text { nutricional dos pacientes } \\
\text { cirróticos por diferentes } \\
\text { métodos: antropometria } \\
\text { (peso, altura, IMC, CB,CMB } \\
\text { e PCT), força de preensão } \\
\text { palmar, bioimpedância e } \\
\text { ASG. Para comparar o estado } \\
\text { nutricional com o consumo } \\
\text { alimentar utilizou-se o escore } \\
\text { de Child-Pugh. }\end{array}$ & $\begin{array}{l}\text { Dos } 25 \text { pacientes avaliados, } \\
68 \% \text { apresentaram cirrose pelo } \\
\mathrm{HCV}, 56 \% \text { dos pesquisados } \\
\text { apresentaram desnutrição segundo } \\
\text { a CB, a força de preensão palmar } \\
\text { e a composição corporal por } \\
\text { bioimpedância. O consumo } \\
\text { médio de calorias, carboidratos, } \\
\text { proteínas e lipídios estavam } \\
\text { dentro do recomendado. No } \\
\text { entanto, o sódio estava acima das } \\
\text { recomendações, } 106 \pm 57,2 \text { mEq, e } \\
\text { foi inversamente associado com o } \\
\text { escore de Child-Pugh (p = } 0,042 \text { ). } \\
\text { A ingestão de alimentos não } \\
\text { apresentou diferença significativa } \\
\text { com o escore de Child-Pugh e o } \\
\text { estado nutricional. Além disso, a } \\
\text { ingestão de alimentos não variou } \\
\text { com o estágio da doença. }\end{array}$ \\
\hline
\end{tabular}

\section{DISCUSSÃO}

Os artigos pesquisados enfatizaram o $\mathrm{HCV}$ como um agente que raramente causa infecção aguda sintomática. Os sintomas são inespecíficos e autolimitados, ea infecção dificilmente édiagnosticada na fase inicial. Aproximadamente 500 casos de infecção aguda por hepatite $\mathrm{C}$ são notificados no Brasil todos os anos. Em geral, a infecção desencadeia um processo degenerativo discreto e progressivo, culminando com fibrose e cirrose hepática anos após a exposição ao agente infeccioso. ${ }^{5}$

Não há um método padrão-ouro capaz de diagnosticar com precisão modificações no estado nutricional de pacientes com hepatite $\mathrm{C}$, entretanto as metodologias mais utilizadas são: antropometria (peso, altura, IMC, dobras cutâneas, CB, CMB), bioimpedância, ASG, avaliação funcional pela força de preensão palmar, métodos bioquímicos e anamnese alimentar. $^{7}$

Geralmente, as doenças hepáticas, especialmente em fase avançada, resultam em grande impacto nutricional. A desnutrição aumenta a atividade de replicação viral do vírus da hepatite $\mathrm{C}$ e associa-se à aceleração do dano hepático. ${ }^{13}$ Por este motivo, pesquisadores têm direcionado suas investigações para o papel desempenhado pela dieta e a influência do estado nutricional na evolução das doenças hepáticas associadas à infecção pelo HCV. ${ }^{14}$

A desnutrição em pacientes com doença hepática possui origem multifatorial, que pode resultar da ingestão insuficiente de alimentos ou de fatores relacionados à digestão e absorção intestinal deficientes. Também pode estar associado à enteropatias perdedoras de proteínas, distúrbios metabólicos e restrições dietéticas constantes. ${ }^{15}$ Port et al., ${ }^{16}$ no estudo que realizaram também verificaram que a desnutrição é frequente em pacientes com hepatite $\mathrm{C}$, devido a ingestão inadequada de energia, proteínas e micronutrientes.

A ingestão inadequada de nutrientes está associada às restrições dietéticas prolongadas, a sensação de saciedade precoce (devido à presença de ascite volumosa), anorexia, náuseas e encefalopatia hepática. A má digestão e má absorção dos nutrientes podem resultar na deficiência de sais biliares, 
crescimento bacteriano intestinal, gastropatia portal hipertensiva, alterações da motilidade intestinal, lesões da mucosa e aumento da permeabilidade intestinal. ${ }^{17}$

Ismail et al. ${ }^{18}$ verificaram que os pacientes com hepatite $\mathrm{C}$ que apresentavam agravamento da doença hepática possuíam maior risco de depleção do estado nutricional. Desta forma, é importantíssimo avaliar o estado nutricional e otimizar a nutrição nesses pacientes. Outro estudo também correlacionou a desnutrição com a gravidade clínica da doença hepática, sendo que a desnutrição proteico-calórica pode ser identificada em todos os estágios clínicos, mas é mais prevalente em fases avançadas desta doença. Além disso, a presença de desnutrição está associada ao aumento do número de complicações e mortalidade a curto e longo prazo. ${ }^{19}$

Fioravante et al. ${ }^{20}$ demonstraram que os pacientes em tratamento com hepatite $\mathrm{C}$ apresentaram uma perda significativa de peso e isso não foi associado a mudanças no gasto de energia, e sim à diminuição significativa na ingestão de energia.

Bruch et al..$^{21}$ no estudo que realizaram observaram que a perda de peso ocorre em estágios mais avançados da doença hepática. A adoção de métodos que possam ser usados para a identificação precoce das mudanças no estado nutricional pode ser de grande valor para um manejo dietético mais adequado, que seja mais eficaz para combater os estágios iniciais de depleção de proteína, contribuindo desse modo para melhorar o prognóstico. Além disso, a porcentagem elevada de gordura corporal observada nos pacientes com hepatite C crônica, pode ser um fator de risco importante para o surgimento de doenças cardiovasculares e outras complicações associadas, além de ter um impacto direto na qualidade de vida dessas pessoas.

A alimentação pode influenciar a função hepática, uma vez que os nutrientes ingeridos são metabolizados principalmente pelo fígado. A dieta para hepatite $\mathrm{C}$ é semelhante às orientações utilizadas para pessoas sem a doença, porém algumas modificações são necessárias para compensar a desordem metabólica. ${ }^{22}$

Cada vez mais os trabalhos apontam a importância do conhecimento do consumo alimentar dos indivíduos em relação à ingestão energética e proteica, de carboidratos, gorduras e fibras. No estudo realizado por Pinto et al., ${ }^{23}$ a ingestão diária de energia foi $100 \mathrm{kcal}$ superior nos homens e $300 \mathrm{kcal}$ inferior do que a média recomendada para mulheres. Este resultado pode estar relacionado à ocorrência de obesidade, sobrepeso e até mesmo desnutrição.

Carreira e Pereira $^{6}$ verificaram que a média da ingestão de carboidratos foi maior que a recomendada. Com relação aos lipídeos não se verificou consumo acima do preconizado. O consumo das fibras revelou claramente que todos os indivíduos que participaram do estudo não consumiram as quantidades preconizadas.

Um estudo recente avaliou o impacto de mudanças no estilo de vida em uma amostra de 154 indivíduos com doença hepática gordurosa não alcoólica (DHGNA) na cidade de Hong Kong, pelo período de 12 meses. Os pacientes foram divididos aleatoriamente em dois grupos, com e sem intervenção. O grupo com intervenção teve consultas periódicas com nutricionista por um ano. Cada indivíduo recebeu um plano alimentar, conforme as recomendações da American Dietetic Association, com ênfase em frutas e legumes, moderado em carboidratos, baixo teor de gorduras e baixo índice glicêmico. Também obtiveram orientações e acompanhamento de um educador físico. Já, o grupo sem intervenção teve orientação médica de rotina, indicação para redução de carboidratos e lipídeos na alimentação, além da realização de atividade física regular. No $12^{\circ}$ mês de estudo foi observada uma maior redução dos desfechos primários da DHGNA nos pacientes do grupo com intervenção. Os níveis séricos de triglicerídeos foram significativamente menores no grupo com intervenção, os quais apresentaram ainda maior redução no peso corporal, no IMC e na circunferência da cintura. Desta forma, $77 \%$ dos pacientes no grupo com intervenção e apenas $30 \%$ no grupo controle tiveram uma perda de peso superior a $3 \%$, e apenas o grupo com intervenção apresentou perda de peso superior a 10\%. Outra avaliação realizada no estudo foi a associação entre a perda de peso e a DHGNA, onde mais de $40 \%$ dos pacientes que obtiveram redução de no mínimo 3\% do peso tiveram uma melhora na DHGNA, e ainda, o grupo com intervenção obteve redução significativa nos níveis de ALT comparado ao grupo controle. ${ }^{24}$

Nunes et al. ${ }^{25}$ a partir do registro alimentar de três dias, analisaram o consumo de calorias, macronutrientes (carboidratos, proteínas e lipídios) e micronutrientes (zinco, ferro, sódio, potássio e cálcio) 
em pacientes com hepatite $\mathrm{C}$. O número médio de calorias foi de $26,4 \pm 8,3 \mathrm{kcal} / \mathrm{kg}$, sendo que, $48 \%$ tiveram um consumo abaixo do recomendado. $\mathrm{O}$ mesmo aconteceu com as proteínas, em que $60 \%$ dos pacientes apresentaram um consumo abaixo do recomendado. A ingestão de carboidratos estava de acordo com as recomendações para a maioria (52\%) dos pacientes, e todos eles tiveram um consumo de lipídios dentro do recomendado. Quanto ao consumo de micronutrientes, o zinco permaneceu dentro das recomendações para $64,2 \%$, a ingestão de ferro estava dentro da normalidade em $100 \%$ dos indivíduos e o potássio estava abaixo do recomendado em 100\% dos indivíduos. O teor de sódio estava acima das quantidades recomendadas em $72 \%$ dos pacientes, e o cálcio abaixo do consumo recomendado em $96 \%$ dos indivíduos.

Gottschall et al. ${ }^{26}$ observaram que a maioria dos pacientes apresentaram baixa ingestão de cálcio, potássio, zinco e vitamina $\mathrm{C}$ e maior ingestão de sódio, ferro, fósforo e colesterol. Além disso, a ingestão média de energia e proteínas foi de $24,6 \mathrm{kcal} / \mathrm{kg}$ e $1,16 \mathrm{~g} / \mathrm{kg}$ de peso corporal, respectivamente.

Santis e Silva et al. ${ }^{11}$ demonstraram que apenas $41 \%$ dos pacientes apresentaram ingestão saudável de alimentos e 59\% uma ingestão não saudável, sendo que vários pacientes apresentaram ingestão insuficiente de calorias $(59 \%)$ e ingestão excessiva de proteína (36\%) e gordura saturada $(63 \%)$.

A recomendação de uma dieta saudável para pessoas com hepatite $\mathrm{C}$, deve levar em consideração a variedade de alimentos de todos os grupos: cereais integrais, vegetais, leite e derivados magros, ovos, carnes magras e leguminosas, oleaginosas e óleos vegetais. A ingestão energética deve ser adequada, mas não excessiva, e fracionada ao longo do dia, a ingestão proteica deve ser suficiente para combater a infecção e para a regeneração do fígado, dar preferência às frutas e verduras principalmente ricas em vitamina $A$ e vitamina $C$ que têm ação antioxidante para combater os radicais livres (enfatizar a variedade e a cor), limitar o alto teor de gordura (saturada e trans) e os alimentos refinados ricos em açúcar e por fim eliminar o álcool para proteger o fígado e praticar alguma atividade, respeitando as limitações físicas. ${ }^{6}$

\section{CONSIDERAÇÕES FINAIS}

Ainda não há um método padrão-ouro capaz de diagnosticar com precisão modificações no estado nutricional de pacientes com hepatite $\mathrm{C}$, pois a retenção hídrica, edema e ascite são comuns nesses pacientes, podendo haver confusões nas estimativas tanto de sobrepeso quanto de desnutrição. Desta forma, recomenda-se a utilização de parâmetros associados para se obter um resultado mais fidedigno, sendo as principais metodologias: antropometria (peso, altura, IMC, dobras cutâneas, CB, CMB), bioimpedância, ASG, avaliação funcional pela força de preensão palmar, métodos bioquímicos e anamnese alimentar. Logo, são necessários mais estudos e validação de protocolos específicos para a avaliação nutricional ser mais precisa e assertiva.

O diagnóstico e a classificação de distúrbios nutricionais nos pacientes com hepatite $\mathrm{C}$ e a relação do estado nutricional com a evolução da hepatopatia não foram ainda completamente esclarecidos. Podese perceber que alguns estudos trazem a classificação de sobrepeso e excesso de gordura abdominal em detrimento de outros estudos que demonstram a prevalência de desnutrição em estágios mais avançados da doença, devido à ingestão inadequada de energia, macronutrientes e micronutrientes.

Enfim, a inflamação do fígado, em pacientes cronicamente infectados pelo HCV evolui de forma progressiva e, assim, déficits nutricionais em menor grau podem não ser identificados. Verificou-se que diversos estudos demonstraram irregularidades em relação à ingestão de macro e micronutrientes. A terapia nutricional é fundamental, sendo de grande importância a investigação do consumo energético diário de macro e micronutrientes para identificar possíveis déficits de nutrientes essenciais na manutenção e recuperação da saúde destes pacientes.

\section{REFERÊNCIAS}

1. WHO. Hepatitis C. 2016. Disponível em: http://www.who.int. sci-hub.cc/mediacentre/factsheets/fs164/en/.

2. Prati D. Transmission of hepatitis $\mathrm{C}$ virus by blood transfusions and other medical procedures: a global review. J Hepatol 2006;45(4):607-16. doi: http://dx.doi.org/10.1016/j. jhep.2006.07.003

3. Nelson PK, Mathers BM, Cowie B, Hagan H, Des Jarlais D, Horyniak D, Degenhardt L. Global epidemiology of hepatitis B and hepatitis $\mathrm{C}$ in people who inject drugs: results of systematic reviews. Lancet 2011;378(9791):571-83. doi: http://dx.doi. 
org/10.1016/S0140-6736(11)61097-0

4. MohdHanafiah K,Groeger J, Flaxman AD, Wiersma ST. Global epidemiology of hepatitis $\mathrm{C}$ virus infection: new estimates of age-specific antibody to HCV seroprevalence. Hepatology 2013;57(4):1333-42. doi: http://dx.doi.org/10.1002/hep.26141.

5. Brasil. Ministério da Saúde. Departamento de DST, Aids e Hepatites Virais. Protocolo Clínico e Diretrizes Terapêuticas para Hepatite C e Coinfecções. Brasília, 2015.

6. Carreira CM,Pereira PC. M. Perfil nutricional e dietético de indivíduos com hepatite C. Semina Cienc Biol Saúde 2011;32(2):143-54. doi: http://dx.doi.org/10.5433/1679$0367.2011 v 32 \mathrm{n} 2 \mathrm{p} 143$

7. Nunes FF,Fernandes SA, Bertolini CM, Rabito EI, Gottschall CBA. Avaliação nutricional do paciente cirrótico: comparação entre diversos métodos. Sci Med 2012;22(1):12-17.

8. Andrade PV, Lameu EB. Espessura do músculo adutor do polegar: um novo indicador prognóstico em pacientes clínicos. Rev Bras Nutr Clin 2007;22(1):28-35.

9. Hiraoka A, Aibiki T, Okudaira T, Toshimori A, Kawamura T, Nakahara H, Suga Y, Azemoto N, Miyata H, Miyamoto Y, Ninomiya T, Hirooka M, Abe M, Matsuura B, Hiasa Y, Michitaka K. Muscle atrophy as pre-sarcopenia in Japanese patients with chronic liver disease: computed tomography is useful for evaluation. J Gastroenterol 2015;50(12):1206-13. doi: http://dx.doi.org/10.1007/s00535-015-1068-x

10. Hayashi F, Matsumoto Y, Momoki C, Yuikawa M, Okada G, Hamakawa E, Kawamura E, Hagihara A, Toyama M, Fujii H, Kobayashi S, Iwai S, Morikawa H, Enomoto M, Tamori A, Kawada N, Habu D. Physical inactivity and insufficient dietary intake are associated with the frequency of sarcopenia in patients with compensated viral liver cirrhosis. Hepatol Res 2013;43(12):1264-75. doi: http://dx.doi.org/10.1111/hepr.12085 11. Santis e Silva VA, Ferraz MLG, Carvalho-Filho R, LanzoniVP, SilvaAEB, SilvaISS.Association of the nutritional profile with histological findings of patients with genotype 1 chronic hepatitis c infection. Arq Gastroenterol 2015;52(4):31520. doi: http://dx.doi.org/10.1590/S0004-28032015000400012

12. Mendes KDS, Silveira RCCP, Galvão CM. Revisão integrativa: Método de Pesquisa parágrafo a incorporação de Evidências na Saúde e na enfermagem. Texto contexto Enfermagem 2008;17(4):758-64

13. Wendland BE. Nutritional guidelines for persons infected with the hepatitis $\mathrm{C}$ virus: a review of the literature. Can J Diet Pract Res 2001;62(1):7-15

14. Manguso F, D’Ambra G, Menchise A, Sollazzo R, D’Agostino L. Effects of an appropriate oral diet on the nutritional status of patients with HCV-related liver cirrhosis: A prospective study. Clin Nutr 2005;24(5):751-9. doi: http://dx.doi.org/10.1016/j. clnu.2005.02.010

15.Sam J, Nguyen GC. Protein-calorie malnutrition as a prognostic indicator of mortality among patients hospitalized with cirrhosis and portal hypertension. Liver Int 2009;29(9):1396402. doi: http://dx.doi.org/10.1111/j.1478-3231.2009.02077.x
16. Port GZ, Oliveira K, Soldera J, Tovo CV. Biochemical nutritional profile of liver cirrhosis patients with hepatocellular carcinoma. Arq Gastroenterol.2014;51(1):10-5. doi: http:// dx.doi.org/10.1590/S0004-28032014000100003

17. Ferreira LG, Anastácio LR, Lima AS, Correia MITD. Desnutrição e inadequação alimentar de pacientes aguardando transplante hepático. Rev Assoc Med Bras 2009;55(4):389-93. doi: http://dx.doi.org/10.1590/S0104-42302009000400011

18. Ismail FW, Khan RA, Kamani L, Wadalawala AA, Shah HA, Hamid SS, Jafri W. Nutritional Status in Patients with Hepatitis C. J Coll Physicians Surg Pak. 2012;22(3):139-42. doi: http:// dx.doi.org/02.2012/JCPSP.139142

19. Teiusanu A, Andrei M, Arbanas T, Nicolaie T, Diculescu M. Nutritional Status in Cirrhotic Patients. Maedica (Buchar) 2012;7(4):284-89

20. Fioravante M, Alegre SM, Marin DM, Lorena SL, Pereira TS, Soares EC. Weight loss and resting energy expenditure in patients with chronic hepatitis $\mathrm{C}$ before and during standard treatment.Nutrition 2012;28(6):630-4. doi: http://dx.doi. org/10.1016/j.nut.2011.08.010

21. Bruch JP, Álvares-da-Silva MR,Alves BC,Dall'Alba V. Reduced hand grip strength in overweight and obese chronic hepatitis C patients.ArqGastroenterol 2016;53(1):31-5. doi: http://dx.doi.org/10.1590/S0004-28032016000100007

22. Okita M. Chronic hepatic disease and dietary instruction. Hepatol Res. 2004;30:92-5. doi: http://dx.doi.org/10.1016/j. hepres.2004.10.012

23. Pinto GS, Zanolla AF, Tovo CV, Gottschall CB, Buss C. Nutritional status and food intake of $\mathrm{HCV} / \mathrm{HIV}$ coinfected patients. NutrHosp 2016;33(5):1123-8. doi: http://dx.doi. org/10.20960/nh.576

24. Wong VW, Chan RS, Wong GL, Cheung BH, Chu WC, Yeung DK, Chim AM, Lai JW, Li LS, Sea MM, Chan FK, Sung JJ, Woo J, Chan HL. Community-based lifestyle modification programme for non-alcoholic fatty liver disease: A randomized controlled trial. J Hepatol 2013;59(3):536-42. doi: http://dx.doi. org/10.1016/j.jhep.2013.04.013

25. Nunes FF, Bassani L, Fernandes AS, Deutrich ME, Pivatto BC, Marroni CA. Food consumption of cirrhotic patients, comparison with the nutritional status and disease staging. Arq Gastroenterol 2016;53(4):250-6. doi: http://dx.doi.org/10.1590/ S0004-28032016000400008

26.Gottschall CB, Pereira TG, Rabito EI, Álvares-Da-Silva MR. Nutritional status and dietary intake in non-cirrhotic adult chronic hepatitis c patients. Arq Gastroenterol 2015;52(3):2049. doi: http://dx.doi.org/10.1590/S0004-28032015000300010

Recebido em:14/02/2018 Aceito em:03/06/2018

Como citar: ALVES, Tauana da Costa; SCHMIDT, Leucinéia; BENETTI, Fábia. Perfil nutricional e dietético de pacientes com hepatite C: uma revisão integrativa da literatura. Revista Interdisciplinar de Promoção da Saúde, Santa Cruz do Sul, v. 1, n. 2, abr. 2018. ISSN 2595-3664. Disponível em: < https://online.unisc.br/seer/index.php/ripsunisc/article/view/11803>. Acesso em: 01 abr. 2018. doi:https://doi.org/10.17058/rips.v1i2.11803 